\title{
Depolarized Photon Correlation Spectroscopy and Shear Stress Relaxation Modulus of Polystyrene
}

\author{
H. L. LIN, Y. S. CHEN, and T. L. YU* \\ Department of Chemical Engineering, Yuan-Ze Institute of Technology, \\ Nei-Li, Taoyuan, Taiwan 32026
}

(Received July 28, 1993)

\begin{abstract}
Depolarized photon correlation spectroscopy (PCS) was applied to investigate polystyrene near the glass transition temperature. The photon correlation function is well described by a single Kohlrausch-Williams-Watts (KWW) function, in which distribution parameters, $b$, is around $0.34-0.38$ which is consistent with the literature results for glass transition. In order to determine the relaxation behavior of polystyrene its depolarized photon correlation function was compared with that from the shear stress relaxation modulus. It appears that relaxation behaviour obtained from depolarized photon correlation spectrum studies is very similar to that of the glass-rubber transition zone in the shear stress relaxation modulus.

KEY WORDS Depolarized Photon Correlation Spectroscopy / Polystyrene / Shear Stress Relaxation Modulus /
\end{abstract}

The dynamic mechanical properties of polymeric glasses are characterized by at least two transitions: the higher temperature corresponds to the transformation from a glass to a rubber state and is often called the $\alpha$-transition; the next highest temperature is $\beta$-transition. In some cases additional low temperature relaxations may be observed and are labelled $\gamma$ and $\delta$-transitions. In order of decreasing temperature these transitions are related to the thermally-activated motion of molecular subgroups containing increasingly smaller numbers of atoms.

Since these molecular motions involve different activation energies (the smaller the molecular sub-group, the lower the activation energy), the separation of the transition temperatures is dependent on the frequency at which mechanical properties are investigated. Also, the process responsible for the higher transition temperature will be the slower molecular motion at any chosen observation temperature. This conclusion can be drawn by considering the increase in energy or temperature needed to speed up the molecular movements matching to the observed time scale or frequency. It comes as no surprise to chemists that the slower process generally has a higher activation energy. This means that when during the joint variation of temperature and frequency, the $\alpha$-process has larger temperature coefficient.

For studying dynamic processes as in different frequency regions, various techniques, such as NMR, dielectric relaxation, depolarized photon correlation spectroscopy (PCS), and viscoelasticity have been used. It is envisaged that different dynamic processes can be physically co-ordinated in a certain way. For a comprehensive understanding of polymer chain dynamics, it is very important to relate the findings of these dynamic processes investigated by different techniques. The investigations described in this work represent ex-

\footnotetext{
* To whom all correspondence should be addressed.
} 
perimental contribution to the characterization of the dynamic mechanical properties of amorphous polymers near the glass transition temperature using photon correlation spectroscopy. The relation between the relaxation behavior of the depolarized photon correlation spectrum and that of the shear stress modulus is discussed.

\section{THEORY}

The time correlation function $C(t)$ of the depolarized Rayleigh light scattering is given by eq $1 .^{1}$

$$
\begin{aligned}
& C(t)= \\
& \quad\left\langle\sum_{i} \sum_{j} \alpha_{y z}^{i}\left[\Omega_{i}(t)\right] \alpha_{y z}^{j}\left[Q_{j}\right] \exp \left(i q \cdot\left(r_{i}(t)-r_{j}\right)\right)\right\rangle
\end{aligned}
$$

In eq 1 the incident light is polarized along the $y$ axis. $\alpha_{y z}\left[\Omega_{i}(t)\right]$ is the $y z$ component of the polarizability tensor of a chemical bond segment $i$, at time $t$ in a laboratory fixed coordinate system. $\Omega_{i}(t)$ is the orientation angle of segment $i$ at position $r_{i}(t)$ at time $t$. It is known that depolarized Rayleigh scattering investigates the collective reorientation motion rather than that associated with single molecules. The collective motion can be expressed in terms of static and dynamic pair correlation between the polarizabilities associated with neighboring molecules. A static pair correlation can be measured from the total intensity measurement. In the polymer systems, the static pair correlation as determined from the depolarized intensity measurement is often expressed in terms of effective optical anisotropy per monomer unit to account for the concentration dependence of the measured total intensity. ${ }^{2}$ If the polymer does not have strong dipolar interactions among segments or there is no symmetry along the chain favoring crystal formation at low temperature, such as polystyrene, segments belonging to different chains do not interact in such a way as to contribute to the static pair correlation.

For entangled polymer, the segmental reorientation rate affects the spectrum through the dependence of $\alpha_{y z}$ on reorientation angles. The segmental position $r_{i}$ with respect to the laboratory frame can be written in terms of the position of center of mass, $R_{I}$, of the entanglement strand- $I,{ }^{3-5}$ to which the segment- $i$ belongs, and the vectot from the center of mass to the segmental position, $r_{i I}$. Similar modification is used for $r_{j}$. With these modifications plus the fact that the size of an entanglement strand (the distance between two adjacent entanglement points is between 30 and $80 \AA$ for hydrocarbon polymers) is much smaller than the scattering wavelength, i.e., $q \cdot r_{i I} \ll 1, C(t)$ can be written as:

$C(t)=$

$\sum_{i j} \sum_{I J}\left\langle\alpha_{y z}^{i, I}\left[\Omega_{i}(t)\right] \alpha_{y z}^{j, J}\left[\Omega_{j}\right] \exp \left(i q \cdot\left(R_{I}(t)-R_{J}\right)\right)\right\rangle$

Consider a homopolymer consisting of long chains, which entangle with one another. In the melt or glass state, one chemical segment comes close to other segments, which either belong to the same chain or other chains. Because of the separation distance and the screening effect of entanglement, it is reasonable to assume that both static and dynamic correlations between chemical segments belonging to different entanglement strands are negligible compared to those within the same entanglement strand. We also assume that the translational motion of the center of mass of an entanglement strand is independent of the segmental reorientation. Then $C(t)$ becomes

$$
\begin{aligned}
& C(t)= \\
& \sum_{I}\left\langle\sum_{i j} \alpha_{y z}^{i, I}\left[\Omega_{i}(t)\right] \alpha_{y z}^{j, I}\left[\Omega_{j}\right]\right\rangle\left\langle\exp \left(i q \cdot\left(R_{I}(t)-R_{I}\right)\right)\right\rangle
\end{aligned}
$$

The characteristics time, $\tau_{I}$, associated with the segmental reorientation correlation func- 
tion $\left\langle\sum_{i j} \alpha_{y z}^{i, I}\left[\Omega_{i}(t) \alpha_{y z}^{j, I}\left[\Omega_{j}\right]\right\rangle\right.$ is much shorter than that of translational motion correlation function $\left\langle\exp \left(i q \cdot\left(R_{I}(t)-R_{I}\right)\right)\right\rangle$. In a short time of the order $\tau_{I}$, because of topological constraint effect due to entanglement, the range that can be covered by the translational motion of the center of mass of an entanglement strand is much smaller than the wavelength of the scattering light. ${ }^{3}$ Thus, $\left\langle\exp \left(i q \cdot\left(R_{I}(t)-R_{I}\right)\right)\right\rangle$ is basically equal to 1 as far as the correlation life time of reorientation is concerned, and the time correlation function $C(t)$ of the depolarized Rayleigh scattering spectrum of a polymer melt becomes

$$
C(t)=\sum_{\mathrm{I}}\left\langle\sum_{i j} \alpha_{y z}^{i, I}\left[\Omega_{i}(t)\right] \alpha_{y z}^{j, \mathrm{I}}\left[\Omega_{j}\right]\right\rangle=\sum_{\mathrm{I}} C_{\mathrm{I}}(t)
$$

In the polymer melt the polymer molecule is treated as a Gaussian chain composed of a number of sub-chain units. Each unit is known as a Kuhn segment which is equivalent to a Rouse segment in viscouse medium. ${ }^{3}$ The polymer molecules is modeled as a chain of freely jointed Kuhn segments within which the chemical segments are correlated dynamically and statically.

Kuhn segments are frely jointed; and thus the pair correlation terms in eq 4 are limited to the chemical segments belonging to the same Kuhn segment. The polarizability tensor $\alpha_{y z}$ of the Kuhn segment can be expressed as the reorientation motion of the Kuhn segment and the reorientation motions of chemical segments with a Kuhn segment

$$
C_{\mathrm{I}}(t)=\langle\beta(t) \beta(0)\rangle\langle P[u(t) \cdot u(0)]\rangle
$$

In eq $5 \beta(t)$ is due to the motion of chemical segments and $u(t)$ is the unit vector representing the direction of the symmetry axis of a Kuhn segment at time $t$.

The rates of reorientation of the Kuhn segment and its chemical segments differ widely in time scales so that during the reorientation of a Kuhn segment, chemical segments inside move independently as though the principal axes of the polarizability tensor of the Kuhn segment are fixed in instantaneous orientation.

It is well known that a stress relaxation spectrum of a nearly monodisperse polymer melt shows three characteristic. regions ${ }^{3,4}$ : the glass-rubber transition region, plateau region, and terminal region. Equation 5 indicates that the depolarized Rayleigh scattering contains mainly two components: one shows the fast motion of the local chemical segments within the Kuhn segment and the other, the slow reorientation motion of the Kuhn segment. In this respect the fast motion is related to the so-called $\beta$-motion, ${ }^{6}$ and the reorientation motion of the Kuhn segment is related to the so-called $\alpha$ motion (the glass-rubber transition) which can be measured by dynamic light scattering. ${ }^{7} \beta$ motion involves only a few chemical segments, while $P[u(t) \cdot u]$ involves the whole Kuhn segment, thus, the activation energy associated with the former should be much smaller than that associated with the latter. The activation energy of the $\alpha$ process is relatively large and becomes larger as the temperature approaches $T_{\mathrm{g}}$; the temperature dependence of the $\alpha$ process is described by the Williams-Landel-Ferry (WLF) equation. ${ }^{8}$ The slow down of reorientation motion of the Kuhn segment can be viewed as corresponding to the stiffening of the Rouse segment. Therefore, the polymer becomes glassy as the correlation time of $P[u(t) \cdot u]$ becomes very long.

There is a temperature range close to the glass-rubber transition, where $P[u(t) \cdot u]$ is slow enough to be observed by PCS, and the Rouse segment is still "soft" enough to maintain its "rubber"-elasticity nature so that the viscoelastic spectrum of the polymer can be measured. In the present work, we show that the relaxation data from depolarized photon correlation spectroscopy are in agreement with stress relaxation data in the glass-rubber transition region. 


\section{EXPERIMENTAL}

\section{Sample Preparation}

Styrene monomer (purchased from Aldrich Chemical Co., Inc.) was purified by vacuum distillation several times very slowly and carefully. The freshly distilled monomer was mixed with $0.05 \%$ by weight of AIBN initiator (previously recrystallized from methanol). The mixture of monomer and initiator was then filtered through a $0.5 \mu \mathrm{m}$ Millipore filter paper several times, and finally filtered directly into a sample cell suitable for the light scattering experiment. The monomer was placed in the sample cell under vacuum for approximately $30 \mathrm{~min}$ and then the cell was sealed under vacuum. To effect polymerization a mixture of monomer and initiator in the sample cell was maintained at $80^{\circ} \mathrm{C}$ for 3 days, $90^{\circ} \mathrm{C}$ for 5 days, $100^{\circ} \mathrm{C}$ for 1 week, and $120^{\circ} \mathrm{C}$ for 1 week sequentially. The sample was then kept around $110^{\circ} \mathrm{C}$ when it was not used.

The apparent glass transition temperature of the polystyrene sample was measured by Perkin-Elmer differential scanning calorimeter at several heating (and cooling) rates of $20^{\circ} \mathrm{C} \min ^{-1}, 10^{\circ} \mathrm{Cmin}^{-1}, 5^{\circ} \mathrm{C} \mathrm{min}^{-1}$, and $2^{\circ} \mathrm{Cmin}^{-1}$. $T_{\mathrm{g}}$ was then determined by extrapolating to zero heating (or cooling) rate and found to be $100.5^{\circ} \mathrm{C}$.

\section{Instrumentation}

The light scattering comprised an argon ion laser source (Lyconix Radiation) providing $300 \mathrm{~mW}$ of power at $514 \mathrm{~nm}$, and a 256channel multi-tau digital correlator (Brookhaven BI2030 AT). To study oreintation fluctuation (or anisotropic fluctuations) the VH configuration of the scattered light was observed. A horizontal polarizer was inserted between the sample cell and PMT detector, so that only horizontally-polarized scattered light was detected.

\section{Data Analysis}

The measured quantity in PCS is the in- tensity correlation function, denoted by $C(t)$. The quantity, $C(t)$, is related to the relaxation spectrum $\Phi(t)$ as:

$$
C(t)=B+A \Phi^{2}(t)
$$

for a homodyne experiment. ${ }^{1,9}$ Where " $A$ " is an instrument constant which depends on the number of coherence areas observed in the sample volume and also depends on the sample time increment, and " $B$ " is a small baseline correction factor in the order of $10^{-3} \cdot{ }^{10}$

Measured intensity correlation functions are normalized by the instrument baseline. This means that proper normalization requires measurement of the autocorrelation function at $t=\infty$. For this reason measurements were made in long duration to permit the full decay of autocorrelation function. However, for polymers near the glass transition temperature, this is not always possible as there may be relaxation times much longer than the longest time available by the correlator. In addition, to get the complete relaxation spectrum, we need to make measurements at times short enough so that the autocorrelation function reaches a constant value. Thus, in practice even at the shortest time of measurement, most correlation functions are still increasing towards the time zero value. Hence in most cases, the temperature range is narrow for keeping the zero time independent and baseline accurate.

In most previous studies on amorphous polymers using a correlator $^{10-29}$ the Kohlrausch-Williams-Watts ${ }^{30,31}$ (KWW) fractional exponential relation of the form

$$
\Phi_{\text {relax }}(t)=\mathrm{e}^{-\left(t / \tau_{0}\right)^{b}}
$$

where $0<b<1$, generally yields an excellent fit to the experimental data. In eq $7, b$ may be interpreted as a width parameter such that the distribution of relaxation times becomes broader as $b$ becomes smaller. The approximate form of the relaxation time distribution has now been determined as a function of $b$ and can be used to interpret the results. 
The average relaxation time of the decay function described by eq 7 may be computed as follows:

$$
\langle\tau\rangle=\int \Phi(t) \mathrm{d} t=\int \mathrm{e}^{-\left(t / \tau_{0}\right)^{b}} \mathrm{~d} t=\frac{\tau_{0}}{b} \Gamma(1 / b)
$$

where $\Gamma$ is the gamma function. Extraction of the parameters for KWW equation is accomplished by using a non-linear least squares fit to the experiment data.

When average relaxation times are plotted in an Arrhenius fashion, i.e., $\log \langle\tau\rangle$ vs. $1 / T$, where $T$ is absolute temperature, attempt was made to fit the data into a WLF expression of the form:

$$
\begin{aligned}
& \langle\tau(T)\rangle= \\
& \left\langle\tau\left(T_{0}\right)\right\rangle \exp \left[-2.303 C_{1}\left(T-T_{0}\right) /\left(C_{2}+T-T_{0}\right)\right]
\end{aligned}
$$

where $T_{0}$ is the reference temperature, $C_{1}$ and $C_{2}$ are adjustable parameters referred to $T_{0}$, and $\tau\left(T_{0}\right)$ is the average relaxation time at $T_{0}$. If a temperature $T_{0}$ other than $T_{\mathrm{g}}$ is chosen as a reference temperature, parameters $C_{1}^{\mathrm{g}}$ and $C_{2}^{\mathrm{g}}$ corresponds to $T_{\mathrm{g}}$ can be calculated from the following relations ${ }^{8}$ :

$$
\begin{gathered}
C_{1}^{\mathrm{g}}=C_{1}^{0} C_{2}^{0} /\left(C_{2}^{0}+T_{\mathrm{g}}-T_{0}\right) \\
C_{2}^{\mathrm{g}}=C_{2}^{0}+T_{\mathrm{g}}-T_{0}
\end{gathered}
$$

In addition, apparent activation energy can be calculated on the basis of these parameters as follows:

$$
\begin{aligned}
E_{\mathrm{a}}= & E\left[\frac{\mathrm{d} \ln \langle\tau\rangle}{\mathrm{d}(1 / T)}\right]= \\
& 2.303 R C_{1}^{0} C_{2}^{0} T^{2} /\left(C_{2}^{0}+T-T_{0}\right)^{2}
\end{aligned}
$$

This quanity $E_{\mathrm{a}}$, instead of being temperature independent, increases rapidly with decreasing temperature and at $T_{\mathrm{g}}$ attains the value

$$
E_{\mathrm{a}}=2.303 R C_{1}^{\mathrm{g}} T_{\mathrm{g}}^{2} / C_{2}^{\mathrm{g}}
$$

\section{RESULTS AND DISCUSSION}

Figure 1 shows the data of the correlation

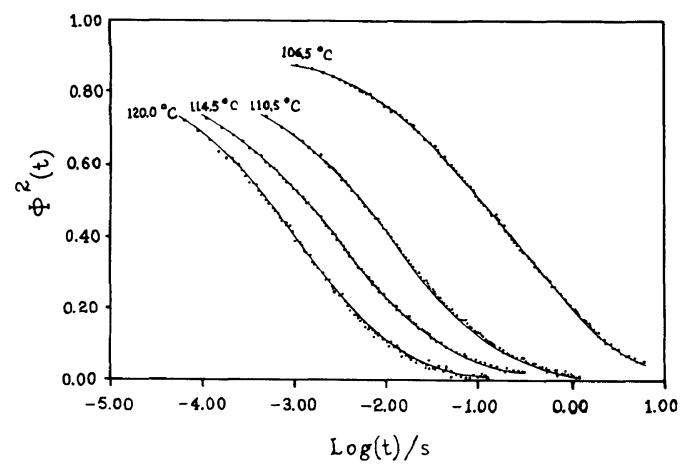

Figure 1. $\Phi^{2}(t) v s . \log t$ of polystyrene at various temperatures.

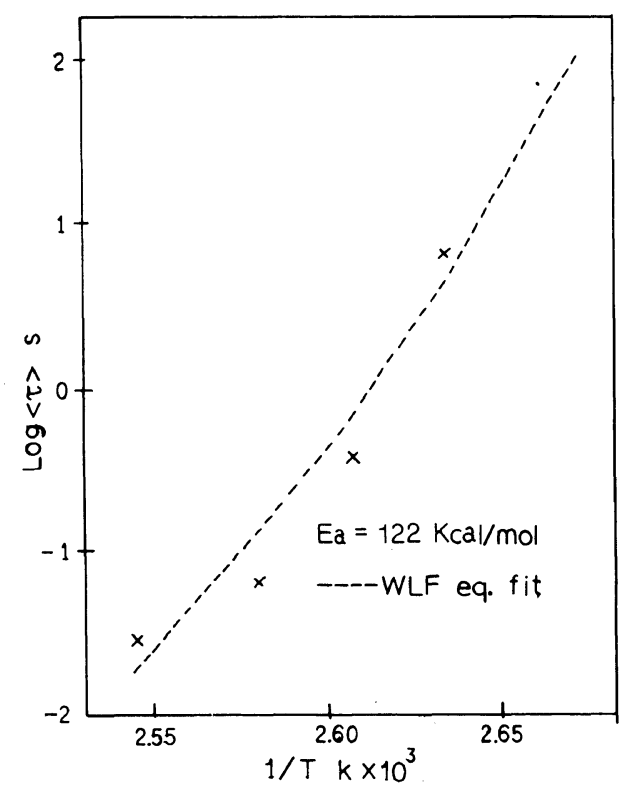

Figure 2. Arrhenius plot of polystyrene. $\log \langle\tau\rangle v s$. $1 / T$.

functions with the curve fitting to a $\mathrm{KWW}$ equation (eq 7) of polystyrene at four different temperatures $106.5^{\circ} \mathrm{C}, 110.5^{\circ} \mathrm{C}, 114.5^{\circ} \mathrm{C}$, and $120.0^{\circ} \mathrm{C}$. Within experimental error this correlation is well represented by KWW equation. The relaxation parameters extracted from these data are summarized in Table I. The distribution parameter, $b$, is found to be in the neighborhood of 0.37 and is independent of temperature, i.e., the correlation functions are highly non-exponential and the width of the 
Table I. Relaxation parameters of polystyrene

\begin{tabular}{lllll}
\hline Temp $/{ }^{\circ} \mathrm{C}$ & 106.5 & 110.5 & 114.5 & 120.0 \\
\hline$A$ & 0.098 & 0.130 & 0.128 & 0.11 \\
$\tau_{0} / \mathrm{s}$ & 1.63 & 0.084 & 0.013 & 0.0060 \\
$b$ & 0.370 & 0.360 & 0.366 & 0.380 \\
$B$ & 0.00080 & 0.00183 & 0.004 & 0.00093 \\
$\langle\tau\rangle / \mathrm{s}$ & 6.71 & 0.38 & 0.060 & 0.025 \\
\hline
\end{tabular}

Table II. WLF parameters of polystyrene

\begin{tabular}{lcccc}
\hline$T_{\mathrm{g}} / \mathrm{K}$ & $C_{1}^{\mathrm{g}}$ & $C_{2}^{\mathrm{g}}$ & Expt. method & Reference \\
\hline 370.7 & 13.3 & 47.5 & Viscous flow & 8,35 \\
370.0 & 11.6 & 27.6 & Compliance & 8,35 \\
374 & 13.3 & 48.2 & PCS & This work \\
373 & 13.0 & 62.77 & PCS & 11,12 \\
\hline
\end{tabular}

distribution of relaxation times remains approximately constant within the temperature range in concern.

Average relaxation times are presented in an Arrhenius plot in Figure 2, from which the apparent activation energy is estimated approximately $122 \mathrm{kcal} \mathrm{mol}^{-1}$.

We fitted $\langle\tau\rangle$ at various temperatures to the WLF equation by choosing $T_{\mathrm{g}}$ as a reference temperature. We obtain $\left\langle\tau\left(T_{\mathrm{g}}\right)\right\rangle=$ $100.0 \mathrm{~s}, C_{1}^{\mathrm{g}}=13.3, C_{2}^{\mathrm{g}}=48.2$. The fit curve of WLF equation (eq 9) is also shown in Figure 2. Table II shows the WLF parameters $C_{1}^{\mathrm{g}}$ and $C_{2}^{\mathrm{g}}$ of polystyrene measured by photon correlation spectroscopy in comparison with the results from viscous flow and transition recoverable compliance. From Table II, it is clear that WLF parameters derived from our PCS measurements are in good agreement with those of other measurements.

Light scattering from bulk polymers occurs as a result of fluctuation in the center of mass position and the orientation of polymeric chain segments. Incident light is typically polarized either vertically $(\mathrm{V})$ or horizontally $(\mathrm{H})$ with respect to the scattering plane. If incident and scattered light are polarized in the vertical direction (VV), the symmetry of the fluctua- tion is longitudinal. $\mathrm{VH}$ (HV) scattering has transverse symmetry. At a $90^{\circ}$ scattering angle, scattered light observed in the VV scattering configuration is a superposition of an isotropic component (due to density fluctuations) and an anisotropic (due to oreintational fluctuations) component, whereas scattered light observed in the $\mathrm{VH}$ configuration is due only to anisotropic fluctuations. Thus, by measuring both the VV and VH scattering spectra, it is possible to determine the isotropic component.

The isotropic component arises from density fluctuations and can be shown closely related to the relaxational behavior of the bulk (compressional) modulus $K$ and shear (stress) modulus $G$ in a combination known as the longitudinal stress modulus $M=K+4 / 3 G$. On the other hand, due to dynamic coupling of the molecular reorientation with the translational motion, ${ }^{1,32}$ the anisotropic component of the scattering spectrum is closely associated with the relaxation behavior of the shear modulus $G^{32}$

It may be of interest to compare our light scattering results with the relaxation of the shear modulus $G(t)$. Aklonis and Tobolsky ${ }^{33}$ measured the shear modulus $G(t)$ of a monodisperse polystyrene sample with molecular weight 267000 . The data were reduced to a temperature $110.0^{\circ} \mathrm{C}$. In analyzing Aklonis and Tobolsky's data in the modulus region $10.0 \mathrm{dyn}^{-2}>\log G(t)>6.0 \mathrm{dyn} \mathrm{cm}^{-2}, \quad \mathrm{a}$ KWW form

$$
G(t)=G_{\mathrm{n}}+\left(G_{\mathrm{g}}-G_{\mathrm{n}}\right) \exp \left[-\left(t / \tau_{\mathrm{G}}\right)^{b_{\mathrm{G}}}\right]
$$

is assumed for the shear modulus $G(t)$, where $G_{\mathrm{g}}$ is the shear modulus at zero time (glassy region) and $G_{\mathrm{n}}$ is the shear modulus at plateau region. Since $G_{\mathrm{g}} \gg G_{\mathrm{n}}$. We may write eq 14 as follows:

$$
G(t)=G_{\mathrm{g}} \exp \left[-\left(t / \tau_{\mathrm{G}}\right)^{\mathbf{b}_{\mathrm{G}}}\right]
$$

In Figure 3, we plot the fit of $G(t)$ to eq 15 and obtained $b_{\mathrm{G}}=0.385$ and $\tau_{\mathrm{G}}=0.0545 \mathrm{~s} . b_{\mathrm{G}}$ and $\tau_{\mathbf{G}}$ for shear modulus relaxation $G(t)$ are 


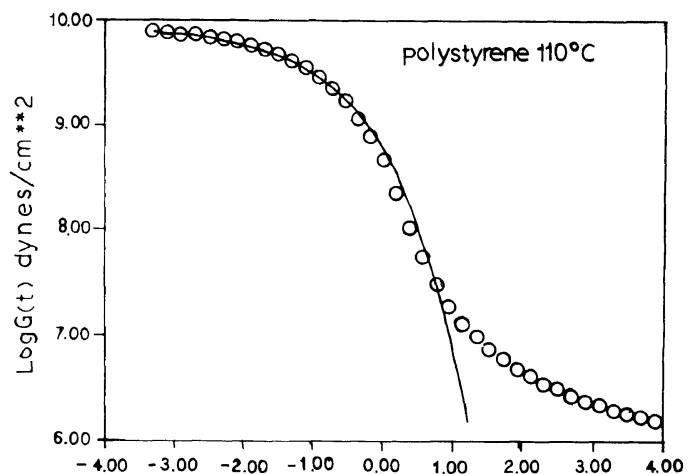

Figure 3. $\log G(t) \quad$ vs. $\log t, \quad b_{\mathrm{G}}=0.385 ; \tau_{\mathrm{G}}=0.0545 \mathrm{~s}$; $\log G_{\mathrm{G}}=9.97 .00000$, data of Aklonis et al. ${ }^{33} ;$ _, curve fitting of eq 15 .

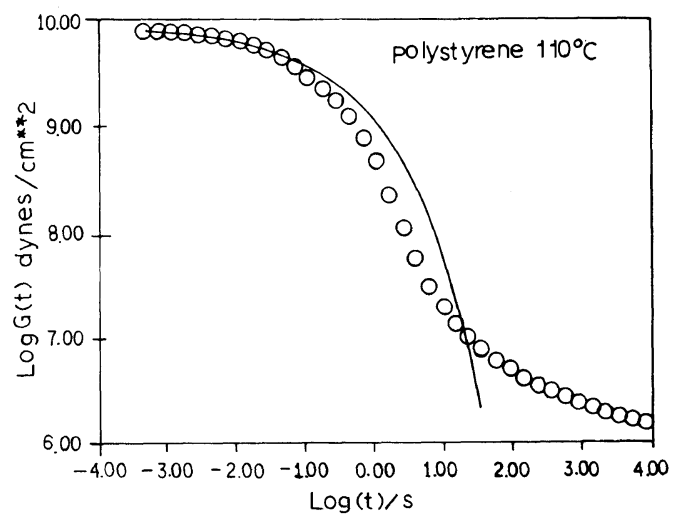

Figure 4. $\log G(t)$ vs. $\log t$. - , light scattering data calculated from eq $15 . b_{\mathrm{G}}=0.360 ; \tau_{\mathrm{G}}=0.084 \mathrm{~s}, \log G_{\mathrm{G}}=$ 9.97. 00000 , mecahnical data of Aklonis et al. ${ }^{33}$

very close to the $b=0.36$ and $\tau_{0}=0.084 \mathrm{~s}$ of our PCS data at $110.0^{\circ} \mathrm{C}$.

Ngai and Plazek ${ }^{34}$ analyzed recoverable shear compliance data of polystyrene with molecular weight of $4.7 \times 10^{4}, 1.89 \times 10^{5}$, $1.22 \times 10^{5}, 6.0 \times 10^{5}, 8.0 \times 10^{5}$, and $3.8 \times 10^{6}$. The time dependent shear modulus $G(t)$ was calculated from shear creep compliance $J(t)$ by the following equation:

$$
\int J(\tau) G(t-\tau) \mathrm{d} \tau=t
$$

Using eq 15 to fit $G(t)$, they obtained $0.35<b_{\mathrm{G}}<0.38$. These results are very close to $b_{\mathrm{G}}=0.385$ of our calculation based on
Aklonis and Tobolsky's measurements and $0.36<b<0.38$ of our PCS data.

In Figure 4, a comparison of the relaxation behavior between the depolarized light scattering and shear modulus $G(t)$ is shown. The circle points are the shear modulus $G(t)$ of polystyrene measured by Aklonis and Tobolsky at $110.0^{\circ} \mathrm{C} . b_{\mathrm{G}}=0.36$ and $\tau_{\mathrm{G}}=0.084$ extracted from our photon correlation function of polystyrene at this temperature and $G_{\mathrm{g}}$ obtained from Aklonis' shear modulus relaxation data were substituted into eq 15 and plotted in solid curve in Figure 4 . The relaxation time of light scattering is longer than the time dependent shear modulus $G(t)$.

From the above analysis, it can be ascertained that the relaxation behavior of depolarized light scattering is very similar to that of the transition zone of shear stress relaxation modulus.

The agreement supports our interpretation that the relaxation time observed from the depolarized Rayleigh scattering can be attributed to the reorientation of a Kuhn segment, which corresponds to the motion of a Rouse segment.

\section{CONCLUSIONS}

The relaxation behavior of polystyrene in the glass transition regime was studied via the depolarized photon correlation spectroscopy. The light observed in the depolarized (VH) scattering configuration is due only to anisotropic fluctuation.

For polystyrene, we found that correlation functions are adequately described by a single KWW (Kohlrausch-Williams-Watts) function. The distribution parameter, $b$, in the range of $0.36-0.38$ is in agreement with reported values for glass transition process.

The results of depolarized light scattering relaxation results of polystyrene were compared with the shear modulus measurements. The relaxation behavior from these two measurements was found to be very close in 
the range $10^{6}<G(t)<10^{10}\left(\right.$ dyn $\left.^{-2}\right)$.

\section{REFERENCES}

1. B. J. Berne and R. Pecora, "Dynamic Light Scattering," John Wiley, New York, 1976.

2. E. W. Fischer and M. J. Dettenmaier, Non-Cryst. Solids, 31, 181 (1978).

3. M. Doi and S. F. Edwards, "The Theory of Polymer Dynamics," Oxford Univ. Press, New York, 1986.

4. Y. H. Lin, Macromolecules, 20, 3080 (1987).

5. M. Doi and S. F. Edwards, J. Chem. Soc., Faraday Trans., 2, 74, 1989: 1802 (1978).

6. N. G. McCrum, B. E. Read, and G. Williams, "Anelastic and Dielectric Effects in Polymer Solids," John Wiley, New York, 1967.

7. A. Donald and E. J. Kramer, J. Polym. Sci., Polym. Phys., 20, 899 (1982).

8. J. D. Ferry, "Viscoelastic Properties of Polymers," 3rd ed, John Wiley, New York, 1980.

9. B. Chu, "Laser Light Scattering," Academic Press, New York, 1975.

10. G. Meier, G. Fytas, and Th. Dorfmuller, Macromolecules, 17, 957 (1984).

11. G. D. Patterson, C. P. Lindsey, and J. R. Stevens, J. Chem. Phys., 70, 643 (1979).

12. C. P. Lindsey, G. D. Patterson, and J. R. Stevens, $J$. Polym. Sci., Polym. Phys. Ed., 17, 1547 (1979).

13. H. Lee, A. M. Jamieson, and R. Smimha, Macromolecules, 12, 329 (1979).

14. H. Lee, A. M. Jamieson, and R. Simha, J. Macromol. Sci.-Phys., B18, 649 (1980).

15. G. D. Patterson, J. R. Stevens, and C. P. Lindsey, J. Macromol. Sci.-Phys., B(18), 641 (1980).

16. A. M. Jamieson, R. Simha, H. Lee, and J. Tribone, Polym. Eng. Sci., 21, 965 (1981).
17. A. M. Jamieson, R. Simha, H. Lee, and J. Tribone, Ann. Y.N. Acad. Sci., 371, 186 (1981).

18. G. Fytas, Th. Dorfmuler, Y. H. Lin, and B. Chu, Macromolecules, 14, 1088 (1981).

19. C. H. Wang, G. Fytas, D. Lilge, and Th. Dorfmuller, Macromolecules, 14, 1363 (1981).

20. G. Fytas, G. Meier, T. Dorfmuller, and A. Patkowski, Macromolecules, 15, 214 (1982).

21. G. Fytas, A. Patkowski, G. Meier, and T. Dorfmuller, Macromolecules, 15, 870 (1982).

22. G. D. Patterson, P. J. Carrol, and J. R. Stevens, J. Polym. Sci., Phys., 21, 605 (1983).

23. G. D. Patterson, P. J. Carroll, and J. R. Stevens, J. Polym. Sci., Phys., 21, 613 (1983).

24. J. Tribone, A. M. Jamieson, and R. Simha, J. Polym. Sci., Polym. Symposium, 71, 231 (1984).

25. G. Fytas and Th. Dorfmuller, J. Polym. Sci., Polym. Physics, 22, 1471 (1984).

26. M. Lee, R. Ferguson, A. M. Jamieson, and R. Simha, Polym. Commun., 26, 66 (1985).

27. C. H. Wang, J-U. Hagenah, G. Meier, G. Fytas, and E. W. Fischer, J. Appl. Phys., 58, 2879 (1985).

28. G. Fytas, C. H. Wang, G. Meier, and E. W. Fischer, Macromolecules, 18, 1492 (1985).

29. U. Murschall, E. W. Fisher, and Ch. Herkt-Maetzky, J. Polym. Sci., Polym. Lett., 24, 191 (1986).

30. G. Williams and D. C. Watts, Trans. Faraday Soc., 66, 80 (1970).

31. K. L. Ngai and G. Fytas, J. Polym. Sci., Polym. Phys., 24, 1683 (1986).

32. C. H. Wang, Mol. Phys., 41, 541 (1980).

33. J. J. Aklonis and A. V. Tobolsky, J. Appl. Phys., 36, 3483 (1965).

34. K. L. Ngai and D. J. Plazek, J. Polym. Sci., B, Polym. Phys., 24, 619 (1986).

35. D. J. Plazek, J. Appl. Phys., 69, 3480 (1965). 